\title{
Antimicrobial peptides with antiprotozoal activity: current state and future perspectives
}

\author{
Laura Giovati ${ }^{1}$, Tecla Ciociola ${ }^{*}, 1$, Walter Magliani ${ }^{1}$ \& Stefania Conti ${ }^{1}$ \\ ${ }^{1}$ Department of Medicine \& Surgery, Laboratory of Microbiology \& Virology, University of Parma, Parma, Italy \\ *Author for correspondence: tecla.ciociola@unipr.it
' antimicrobial peptides (AMPs) have been hypothesized as a possible alternative or complement to conventional antiprotozoal drugs because of their different mechanisms of action, less likely occurrence of resistance, broad-spectrum activity and generally low toxicity"

First draft submitted: 12 September 2018; Accepted for publication: 10 October 2018; Published online: 30 November 2018

Keywords: antimicrobial peptides • antiprotozoal peptides • Cryptosporidium • drug discovery • killer peptide • leishmania • Plasmodium • protozoal diseases • toxoplasma • trypanosoma

Protozoal infections are responsible for significant morbidity and mortality in humans. To date, no safe and effective vaccine is available against any human protozoal disease. Measures to reduce the burden of protozoal infections are limited to drug treatment of affected patients and vector surveillance and control programs for vector-borne diseases [1]. However, these interventions have led to the selection of both resistant protozoa and vectors. Resistance to several first-line drugs is also rising in nonvector-transmitted protozoa, such as Toxoplasma gondii and pathogenic intestinal protozoa [2]. Moreover, many relevant antiprotozoal drugs need long-term treatment and are often associated with serious adverse effects and high toxicity [3].

In this scenario, bioactive antimicrobial peptides (AMPs) have been hypothesized as a possible alternative or complement to conventional antiprotozoal drugs because of their different mechanisms of action, less likely occurrence of resistance, broad-spectrum activity and generally low toxicity [4].

\section{Mechanisms of antiprotozoal activity}

Studies on the antimicrobial activity of natural and synthetic AMPs have focused mainly on bacteria and fungi [5,6]. Fewer reports exist regarding the effects of AMPs on protozoa and the majority of these involve Plasmodium, Leishmania and Trypanosoma species [4,7,8]. Nevertheless, a growing number of recent studies identified peptide lead candidates for the development of drugs against other protozoal parasites, such as Cryptosporidium parvum and T. gondii.

The prevailing mechanisms of action of the described natural and synthetic antiprotozoal peptides generally involve the interaction with cell membrane. Most of the membrane-active peptides act by perturbation of membrane integrity and induction of osmotic cell lysis. Other membrane-active peptides modify the fluidity of protozoal membranes, impairing the activity of membrane-bound proteins [8]. Anionic phospholipids on the outer membrane of the target cell are responsible for the relative specificity of cationic AMPs toward protozoal over human host cells. Upon infection, intracellular protozoa may induce significant changes in host cell membranes. As an example, Plasmodium falciparum causes alteration in membranes of infected erythrocytes, whose composition gets closer to that of protozoa. This mechanism accounts for the selective interaction of infected erythrocytes with some small cationic AMPs showing an antimalarial activity, such as NK-2 derivative of mammalian NK-lysin [7].

Another mechanism of action of antiprotozoal AMPs involves their translocation into the cell and interaction with intracellular targets, causing the collapse of metabolic and bioenergetic pathways. Histatin 5, a human salivary AMP, is a cell-penetrating peptide that targets mitochondrial ATP synthesis in Leishmania, causing cell death by bioenergetics exhaustion [7]. Recently, crotamine from Crotalus durissus terrificus (tropical rattlesnake) venom was shown to penetrate $P$. falciparum cells, and compromise parasite metabolism by disruption of acidic compartments 
$\mathrm{H}^{+}$homeostasis. A selective internalization of crotamine into P. falciparum infected erythrocytes was also observed, suggesting this AMP as a promising lead molecule for the development of new potential peptidomimetics [9].

Some AMPs are known to induce protozoal death by triggering autophagic- or apoptotic-like processes in Leishmania spp. and Trypanosoma cruzi [7,10]. Recently, following exposition to different AMPs, necrotic- and apoptotic-like cell deaths were observed in T. cruzi and T. gondii, respectively $[11,12]$.

AMPs may interact with multiple targets, exploiting more than one mechanism to kill different developmental stages of a single protozoal species. For example, melittin from bee venom was shown to kill T. cruzi epimastigotes and trypomastigotes by induction of autophagic or apoptotic cell death, respectively [10]. AMPs may also kill diverse protozoa by different mechanisms. An example is the discovery of a novel antiprotozoal activity of the synthetic decapeptide KP. This peptide derives from a single-chain recombinant anti-idiotypic antibody mimicking a wide-spectrum killer toxin produced by Wickerhamomyces anomalus, a yeast that may also be found as symbiont of Leishmania vectors [12,13]. In Leishmania spp. KP targets superficially expressed $\beta$-1,3-glucan molecules, triggering autophagic cell degeneration. However, KP kills T. gondii tachyzoites, where these glucans have not been described, by induction of an apoptosis-like cell death [12].

Although the molecular targets of some antiprotozoal AMPs still need to be elucidated, the broad spectrum of antimicrobial activity, together with the possibility of selection or modification of peptides to target-desired biological pathways, render AMPs attractive compounds for the development of new antiprotozoal drugs.

\section{Issues \& trends in antiprotozoal peptide drug development}

Despite the considerable potential of AMPs as drug candidates, some limitations must be considered for their development and commercial application. The main obstacles so far identified for peptide drug development are the high production costs, as compared with small drugs [14]. However, new strategies of synthesis and lowered monomer costs have markedly increased the interest in developing peptide drugs. As pharmaceutical ingredients, natural peptides present also intrinsic biological weaknesses, including low oral bioavailability, poor chemical and physical stability under physiological conditions and a short circulating plasma half-life [14]. Beside these general issues, the development of peptide-based antiprotozoal drugs presents some specific challenges related to the complex life cycles of protozoa, which often involve multiple stages with significant differences in metabolism, protein expression and membrane composition. Environmentally transmitted protozoa have the ability to form cysts impermeable to water soluble agents, Trypanosomatidae may produce a protective glycocalyx, and Leishmania spp. possess membrane-bound proteases. Moreover, some protozoa present an intracellular stage in the vertebrate host, implicating the requirement of intracellular activity of AMPs or their ability to cooperate with the host defense mechanisms for the elimination of the parasite.

Some of these general and specific aspects have been successfully addressed through rational design approaches for peptide optimization, based on the study of the relationships between amino acid sequence, 3D structure, activity and target spectrum of AMPs [14,15]. Emerging technologies for innovative peptide drugs development combine rational design and in silico structural bioinformatics approaches for the identification of novel peptide inhibitors based on the sequences of protozoal critical proteins that are conserved in the parasites, but differ in mammalian orthologous proteins. This approach recently led to the identification of a peptide therapeutic lead, able to inhibit kinase receptors of Leishmania and Trypanosoma, that impaired growth of both parasites in vitro and in vivo without toxic effects [16]. In another work, 3D structure and molecular docking studies were implemented to design inhibitory peptides, targeting T. gondii RON4-human $\beta$-tubulin interactions, that were effective in preventing host invasion [17].

In silico approaches to identify new classes of antiprotozoal peptides may also be integrated with transcriptome studies to discover novel molecules for the control of protozoal diseases. Transcriptome analysis may unravel the gene expression profile during infection, as well as provide information on the physiology and mechanism of action of parasitic molecules, allowing the identification of strategic target proteins for the design of novel AMPs. Transcriptomes from different developmental stages of protozoa exposed to AMPs may also be investigated to identify potential drug targets and vaccine candidates [4].

Another challenging approach for innovative peptide drugs involves the development of drug-delivery systems where the bioactive peptide is grafted to a carrier molecule, such as an antibody or a cell-penetrating peptide. As an example, recombinant molecules combining monoclonal antibodies targeting $C$. parvum fused to bioactive peptides proved to efficiently kill $C$. parvum sporozoites in vitro and inhibit infection when administered orally in a prophylactic murine model of cryptosporidiosis [18]. Moreover, cell-penetrating peptides may be exploited to ferry 
a wide variety of effectors, including other peptides, across the protozoal cell membrane. Pyrrochoricin, a short insect-derived proline-rich AMP, has shown ability to deliver a peptide cargo in C. parvum. Previous experiments have shown that this peptide is also capable of transduction across mammalian cells with limited cytotoxicity, demonstrating the potential to target $C$. parvum within intestinal epithelial cells [19].

Biotechnological approaches have also been proposed for the production of transgenic vectors expressing antiparasitic AMPs to block disease transmission. A different biotechnological approach relies on paratransgenic systems, based on symbionts able to colonize the insect vector of protozoal diseases transformed to express antiparasitic AMPs [20].

\section{Conclusion}

In conclusion, diverse natural and synthetic AMPs have been tested for their activity against medically relevant protozoa and some have displayed potential as candidates for the development of new peptide-based approaches against protozoal diseases. A better understanding of targets, mechanisms of action, and pharmacokinetics properties of known antiprotozoal AMPs is needed to ascertain the most potent and selective structural variants. Additionally, the finding of new targets from genome and transcriptome analyses and the development of high-throughput screening platforms may advance the design and discovery of new effective antiprotozoal peptides.

\section{Financial \& competing interests disclosure}

The authors have no other relevant affiliations or financial involvement with any organization or entity with a financial interest in or financial conflict with the subject matter or materials discussed in the manuscript apart from those disclosed.

No writing assistance was utilized in the production of this manuscript.

\section{References}

1. Sacks DL. Vaccines against tropical parasitic diseases: a persisting answer to a persisting problem. Nat. Immunol. 15(5), 403-405 (2014).

2. El-Taweel HA. Understanding drug resistance in human intestinal protozoa. Parasitol. Res. 114(5), 1647-1659 (2015).

3. Rosenthal PJ. Antiprotozoal drugs. In: Basic and Clinical Pharmacology (13th Edition). Katzung BG, Trevor AJ (Eds). McGraw-Hill Medical, NY, USA, Chapter 52 (2015).

4. Lacerda AF, Pelegrini PB, De Oliveira DM, Vasconcelos ÉAR, Grossi-De-Sá MF. Anti-parasitic peptides from arthropods and their application in drug therapy. Front. Microbiol. 7, 91 (2016).

5. Ageitos JM, Sánchez-Pérez A, Calo-Mata P, Villa TG. Antimicrobial peptides (AMPs): ancient compounds that represent novel weapons in the fight against bacteria. Biochem. Pharmacol. 133, 117-138 (2017).

6. Ciociola T, Giovati L, Conti S, Magliani W, Santinoli C, Polonelli L. Natural and synthetic peptides with antifungal activity. Future Med. Chem. 8(12), 1413-1433 (2016).

7. Pretzel J, Mohring F, Rahlfs S, Becker K. Antiparasitic peptides. In: Yellow Biotechnology I: Insect Biotechnologie in Drug Discovery and Preclinical Research, Vilcinskas A (Ed.), Springer, Berlin, Heidelberg, Germany, 157-192 (2013).

8. Torrent M, Pulido D, Rivas L, Andreu D. Antimicrobial peptide action on parasites. Curr. Drug Targets 13(9), 1138-1147 (2012).

9. El Chamy Maluf S, Dal Mas C, Oliveira EB et al. Inhibition of malaria parasite Plasmodium falciparum development by crotamine, a cell penetrating peptide from the snake venom. Peptides 78, 11-16 (2016).

10. Adade CM, Oliveira IRS, Pais JAR, Souto-Padrón T. Melittin peptide kills Trypanosoma cruzi parasites by inducing different cell death pathways. Toxicon 69, 227-239 (2013).

11. Bandeira ICJ, Bandeira-Lima D, Mello CP et al. Antichagasic effect of crotalicidin, a cathelicidin-like vipericidin, found in Crotalus durissus terrificus rattlesnake's venom gland. Parasitology 145(8), 1059-1064 (2017).

12. Giovati L, Santinoli C, Mangia C et al. Novel activity of a synthetic decapeptide against Toxoplasma gondii tachyzoites. Front. Microbiol. 9, 753 (2018).

13. Martin E, Bongiorno G, Giovati L et al. Isolation of a Wickerhamomyces anomalus yeast strain from the sandfly Phlebotomus perniciosus, displaying the killer phenotype. Med.Vet. Entomol. 30(1), 101-106 (2016).

14. Fosgerau K, Hoffmann T. Peptide therapeutics: current status and future directions. Drug Discov. Today 20(1), 122-128 (2015).

15. Rivas L, Luque-Ortega JR, Andreu D. Amphibian antimicrobial peptides and protozoa: lessons from parasites. Biochim. Biophys. ActaBiomembranes 1788(8), 1570-1581 (2009).

16. Qvit N, Schechtman D, Pena DA et al. Scaffold proteins LACK and TRACK as potential drug targets in kinetoplastid parasites: development of inhibitors. Int. J. Parasitol. Drugs Drug Resist. 6(1), 74-84 (2016).

17. Vetrivel U, Nagarajan H, Thirumudi I. Design of inhibitory peptide targeting Toxoplasma gondii RON4-human $\beta$-tubulin interactions by implementing structural bioinformatics methods. J. Cell. Biochem. 119(4), 3236-3246 (2018). 
18. Imboden M, Riggs MW, Schaefer DA, Homan EJ, Bremel RD. Antibodies fused to innate immune molecules reduce initiation of Cryptosporidium parvum infection in mice. Antimicrob. Agents Chemother. 54(4), 1385-1392 (2010).

19. Boxell A, Lee SHC, Jefferies R et al. Pyrrhocoricin as a potential drug delivery vehicle for Cryptosporidium parvum. Exp. Parasitol. 119(2), 301-303 (2008).

20. Hurwitz I, Fieck A, Read A et al. Paratransgenic control of vector borne diseases. Int. J. Biol. Sci. 7(9), 1334-1344 (2011). 